\title{
REDUCIBILITY VERSUS DEFINABILITY FOR PSEUDOVARIETIES OF SEMIGROUPS
}

\author{
J. ALMEIDA AND O. KLÍMA
}

\begin{abstract}
It is easy to show that a pseudovariety which is reducible with respect to an implicit signature $\sigma$ for the equation $x=y$ can also be defined by $\sigma$-identities. We present several negative examples for the converse using signatures in which the pseudovarieties are usually defined. An ordered example issue from the extended Straubing-Thérien hierarchy of regular languages is also shown to provide a positive example for the inequality $x \leq y$.
\end{abstract}

\section{INTRODUCTION}

Drawing motivation and problems from theoretical computer science, specially from the theory of finite automata and regular languages, the study of finite semigroups has led to substantial developments since the 1960's. The connections between the two areas were formalized in seminal work of Eilenberg [18, 19] where, in particular, the relevant classification of finite semigroups that emerged is in terms of the so-called pseudovarieties. Indeed, through Eilenberg's correspondence, pseudovarieties of semigroups are associated, via syntactical recognition, to classes of languages (varieties) with natural closure properties. Several combinatorial operations on varieties of languages have been shown to correspond to algebraic constructions on pseudovarieties of semigroups and the general aim is to decide membership in a variety by deciding membership in the corresponding pseudovariety. Running through this general program, pseudovarieties of semigroups are often defined as smallest pseudovarieties generated by a given class of semigroups, constructed by applying some algebraic operator on semigroups from given pseudovarieties. While this process does not in general preserve decidability of the membership problem [1, 14, the search for stronger hypotheses on the given pseudovarieties to guarantee decidability for the resulting pseudovariety seems a worthwhile endeavor. Many works in this direction have been developed, starting with deep results of Ash 13 , on the pseudovariety of all finite groups and various attempts of extending it [3, 10, more or less successful depending on the algebraic operator under consideration. The case of the semidirect product was initially based on a result [12] in whose proof a gap was found and which remains to be filled (see the discussion in [28, Chapter 3]).

In the approach considered in [10, several properties of pseudovarieties of semigroups are considered depending on an enriched algebraic signature for

2010 Mathematics Subject Classification. Primary 20M07.

Key words and phrases. pseudovariety, relatively free profinite semigroup, ordered semigroup, completely regular semigroup, commutative semigroup, group. 
finite semigroups given by an implicit signature: besides multiplication, a set of other operations commuting with homomorphisms is taken into account. A basic property is whether the signature is sufficiently rich to define the pseudovariety (definability). Another important property, which is related with the work of Ash [13, is whether the signature is sufficient to witness solutions, modulo the given pseudovariety, of systems of equations with constraints in finite semigroups (reducibility). Even for the system of equations consisting of the single equation $x=y$, where $x$ and $y$ are variables, it is easy to show that reducibility implies definability [10]. Prior to this work, nowhere in the literature seems to be an example showing that the converse does not hold. Many pseudovarieties have been shown to be reducible [6, 17, 7] with respect to the signature in which they are naturally defined, but proofs are very much dependent on the properties of the specific pseudovarieties.

The aim of this paper is to understand the relationship between definability and reducibility. We show, that for simple pseudovarieties of semigroups usually defined within a certain signature, reducibility also holds. In contrast, we present several negative examples, of pseudovarieties which are definable in a natural signature but not reducible with respect to it. The examples are drawn from three natural classes of semigroups: commutative semigroups, groups, and completely regular semigroups. The technique to establish the negative results involves choosing a suitable regular language for which the syntactic congruence is tight enough to have simple to handle classes and allow a combinatorial analysis of the desired witnesses.

\section{Preliminaries}

The reader is referred to standard references [2, 5, 23, 28, for background on semigroups, pseudovarieties, and profinite semigroups. Most of what we write about semigroups may equally well be established for monoids but we usually stick with semigroups. We also consider the extension of the theory of pseudovarieties of semigroups to ordered semigroups [25].

Given a pseudovariety of (ordered or not) semigroups $\mathrm{V}$, the pro- $\mathrm{V}$ semigroup freely generated by a set $A$ is denoted $\bar{\Omega}_{A} \mathrm{~V}$. The pseudovarieties of all finite semigroups and of all finite monoids are denoted, respectively, $\mathrm{S}$ and $\mathrm{M}$.

An implicit signature is a set in which each element belongs to some free profinite semigroup $\bar{\Omega}_{A} \mathrm{~S}$, where $A$ is a finite set, including binary multiplication. The elements $w$ of $\bar{\Omega}_{A} S$ may be seen as $A$-ary (implicit) operations with a natural interpretation $w_{S}: S^{A} \rightarrow S$ on each profinite semigroup $S$ : for each function $\varphi: A \rightarrow S$, we put $w_{S}(\varphi)=\hat{\varphi}(w)$, where $\hat{\varphi}$ is the unique extension of $\varphi$ to a continuous homomorphism $\bar{\Omega}_{A} \mathrm{~S} \rightarrow S$, the existence of which amounts to the universal property defining free profinite semigroups. Even when restricted to finite semigroups, this interpretation is injective and produces exactly those operations that commute with homomorphisms. In particular, for an implicit signature $\sigma$, finite semigroups and $\bar{\Omega}_{A} S$ are naturally viewed as $\sigma$-algebras and this is always the structure of $\sigma$-algebras that we will consider on them. The $\sigma$-subalgebra of $\bar{\Omega}_{A} \mathrm{~S}$ generated by the set $A$ of free generators, denoted $\Omega_{A}^{\sigma} \mathrm{S}$, is easily seen to be the free $\sigma$-algebra in the Birkhoff variety of $\sigma$-algebras generated by $\mathrm{S}$. The elements of $\Omega_{A}^{\sigma} \mathrm{S}$ 
are called $\sigma$-words. Note that, when $\sigma$ is reduced to binary multiplication, $\sigma$-words are simply words, meaning elements of the free semigroup $A^{+}$. In general, elements of $\bar{\Omega}_{A} \mathrm{~S}$ are called pseudowords. The set $A^{+}$is topologically dense in the metric space $\bar{\Omega}_{A} \mathrm{~S}$, that is, every pseudoword is the limit of some sequence of words.

Some relevant and frequently encountered examples of pseudowords may be described as follows: $x^{\omega+k}=\lim _{n \rightarrow \infty} x^{n !+k}(k \in \mathbb{Z})$ and $x^{p^{\omega}}=\lim _{n \rightarrow \infty} x^{p^{n !}}$. These are just a few examples of pseudowords in one variable, of which there are uncountably many. More precisely, let $\hat{\mathbb{N}}$ denote the profinite completion of the semiring $(\mathbb{N},+, \cdot)$ of non-negative integers. The "logarithmic" mapping $\bar{\Omega}_{\{x\}} \mathrm{M} \rightarrow \hat{\mathbb{N}}$ sending the generator $x$ to 1 extends uniquely to an isomorphism of $\bar{\Omega}_{\{x\}} \mathrm{M}$ with the additive semigroup of the profinite semiring $\hat{\mathbb{N}}$. Composition in $\bar{\Omega}_{\{x\}} \mathrm{M}$, given by $(u \circ v)_{S}=u_{S} \circ v_{S}$ "logarithmically" translates to multiplication in $\hat{\mathbb{N}}$. Note that the pseudoword $x^{\omega}$ corresponds to the only nonzero additive idempotent of $\hat{\mathbb{N}}$. The additive semigroup ideal it generates is a subsemiring isomorphic with the profinite completion $\hat{\mathbb{Z}}$ of the usual ring of integers $(\mathbb{Z},+, \cdot)$, under an isomorphism sending $\omega+1$ to 1 ; it should therefore lead to no confusion to abuse notation and denote the inverse isomorphism by $\gamma \mapsto \omega+\gamma$.

Abusing notation, we will also denote by the same symbols the implicit signatures consisting of pseudowords determined by a set of exponents in $\hat{\mathbb{N}}$ and binary multiplication. For instance, we write $\omega=\left\{{ }_{-}{ }^{\omega},{ }_{-}{ }_{-}\right\}$. In case it is stated that $\gamma \in \hat{\mathbb{Z}}$, the notation is interpreted as $\gamma=\left\{{ }_{-}{ }^{\omega+\gamma},{ }_{-} \cdot{ }_{-}\right\}$.

By a pseudoidentity we mean a formal equality $u=v$ of pseudowords $u, v \in \bar{\Omega}_{A} \mathrm{~S}$ for some finite set $A$. In case $u, v \in \Omega_{A}^{\sigma} \mathrm{S}$, we call $u=v$ a $\sigma$-identity. A finite semigroup $S$ satisfies the pseudoidentity $u=v$ and we write $S \models u=v$ if $u_{S}=v_{S}$; this notion and notation are extended to classes of finite semigroups and sets of pseudoidentities by requiring that every semigroup in the class satisfy every pseudoidentity in the set. Sometimes, it is convenient to use the abbreviation $u=1$ to stand for the pair of pseudoidentities $u x=u=x u$, where $x$ does not occur in $u$. The class of all finite semigroups that satisfy all pseudoidentities in a given set $\Sigma$ of pseudoidentities is denoted $\llbracket \Sigma \rrbracket$ and is easily seen to be a pseudovariety. In fact, every pseudovariety is of this form [27]. Pseudoinequalities and satisfaction by finite ordered semigroups are defined similarly. Pseudovarieties of ordered semigroups are also defined by pseudoinequalities [22, 25]. A pseudovariety that may be defined by $\sigma$-identities is said to be $\sigma$-equational or simply equational in case $\sigma$ consists only of binary multiplication.

We recall some notions, simplified to the context that interests us here. They are taken from [10, a paper to which the reader is also referred for further motivation. Given a pseudovariety $\mathrm{V}$, a finite semigroup $S$, elements $s, t \in S$, and an onto continuous homomorphism $\varphi: \bar{\Omega}_{A} \mathrm{~S} \rightarrow S$, by a $\mathrm{V}$-solution of the equation $x=y$ for the triple $(S, s, t)$ we mean a pair $u, v \in \bar{\Omega}_{A} \mathrm{~S}$ such that $\mathrm{V} \models u=v, \varphi(u)=s$, and $\varphi(v)=t$. For an implicit signature $\sigma$, the pseudovariety $\mathrm{V}$ is said to be $\sigma$-reducible (for the equation $x=y)$ if, whenever there is a $\mathrm{V}$-solution for a triple $(S, s, t)$, there is some $\mathrm{V}$-solution consisting of $\sigma$-words. This property is independent of the chosen 
onto continuous homomorphism $\varphi: \bar{\Omega}_{A} S \rightarrow S$ (cf. [10, Proposition 4.1]). In case $\sigma$ is reduced to binary multiplication, we call word reducible a $\sigma$ reducible pseudovariety. Similar notions may be considered for pseudovarieties of ordered semigroups by replacing the equation $x=y$ by the inequality $x \leq y$ and the condition $\mathrm{V} \models u=v$ by $\mathrm{V} \models u \leq v$.

It is easy to see that, if a pseudovariety $\mathrm{V}$ is $\sigma$-reducible, then it is $\sigma$ equational (see [10, Proposition 4.2] for the unordered case, the ordered case being handled similarly).

\section{Positive examples}

In this section, we exhibit some examples of pseudovarieties that are usually defined by $\sigma$-identities that turn out also to be $\sigma$-reducible.

The simplest example is that of locally finite pseudovarieties $\mathrm{V}$, in which, for each positive integer $n$, there is a bound on the size of $n$-generated members of $\mathrm{V}$, that is, $\bar{\Omega}_{A} \mathrm{~V}$ is finite for every finite set $A$. Such pseudovarieties are clearly equational, being defined for instance by all word identities describing the multiplication of a word representative of each element by each generator in each semigroup $\bar{\Omega}_{A} \vee$ with $A$ finite. The following result may be considered a simple exercise and is presented here as a warmup.

Proposition 1. Every locally finite pseudovariety is word reducible.

Proof. Consider a finite semigroup $S, s, t \in S$, and a continuous homomorphism $\varphi: \bar{\Omega}_{A} S \rightarrow S$. Assume that the pair $(u, v)$ is a $\mathrm{V}$-solution of the equation $x=y$ for the triple $(S, s, t)$. Let $\left(u_{n}\right)_{n}$ and $\left(v_{n}\right)_{n}$ be sequences of words converging to the pseudowords $u$ and $v$, respectively. Let $\psi: \bar{\Omega}_{A} \mathrm{~S} \rightarrow \bar{\Omega}_{A} \mathrm{~V}$ be the natural projection, mapping each generator to itself. Since the topologies considered in $S$ and $\bar{\Omega}_{A} \mathrm{~V}$ are discrete, for all sufficiently large $n$, we have $\varphi\left(u_{n}\right)=\varphi(u), \psi\left(u_{n}\right)=\psi(u)$, and similarly for $v_{n}$ and $v$. As $\mathrm{V} \models u=v$ if and only if $\psi(u)=\psi(v)$, it follows that the pair $\left(u_{n}, v_{n}\right)$ is a $\mathrm{V}$-solution of the equation $x=y$ for the triple $(S, s, t)$ whenever $n$ is large enough.

The argument of the preceding proof may be similarly applied to handle arbitrary systems of equations. Except for the fact that only a special type of systems, determined by finite directed graphs, were considered in [10], a much stronger result is [10, Theorem 4.18].

The pseudovariety $\mathrm{J}^{+}=\llbracket 1 \leq x \rrbracket$ corresponds to the one-half level in the Straubing-Thérien hierarchy [26, Proposition 8.4]1] Since the basis is equational, our goal is to prove that the pseudovariety is word-reducible (for $x \leq y$ ). First we must recall that $u \leq v$ is satisfied in $\mathrm{J}^{+}$if and only if every finite subword of $u$ is also a subword of $v$. More precisely, if we define, for any pseudoword $w \in \bar{\Omega}_{A} \mathrm{M}$, the set of all finite $\operatorname{subwords} \operatorname{Sub}(w)$ in the following way

$$
\begin{aligned}
\operatorname{Sub}(w)=\left\{a_{1} a_{2}\right. & \ldots a_{n} \in A^{*} \mid a_{1}, a_{2}, \ldots, a_{n} \in A, \\
& \left.\exists w_{0}, w_{1}, \ldots, w_{n} \in \bar{\Omega}_{A} \mathrm{M}: w=w_{0} a_{1} w_{1} a_{2} \ldots a_{n} w_{n}\right\},
\end{aligned}
$$

then we have $\mathrm{J}^{+} \models u \leq v$ if and only if $\operatorname{Sub}(u) \subseteq \operatorname{Sub}(v)$.

\footnotetext{
${ }^{1}$ The syntactic order that we consider for a language $L \subseteq A^{+}$is $u \leq_{L} v$ if, for all $x, y \in A^{*}, x u y \in L$ implies $x v y \in L$. Some authors [24] consider the opposite order, which naturally leads to reversed pseudoinequalities.
} 
Lemma 2. Given a finite monoid $M$, a homomorphism $\varphi: \bar{\Omega}_{A} \mathrm{M} \rightarrow M$ and a pseudoword $w \in \bar{\Omega}_{A} \mathrm{M}$, there exists a finite subword $v$ of $w$ such that $\varphi(v)=\varphi(w)$.

Proof. There is a sequence of words $\left(w_{n}\right)_{n}$ converging to $w$ in $\bar{\Omega}_{A} \mathrm{M}$ such that $\varphi\left(w_{n}\right)=\varphi(w), n \geq 1$. We consider the Cayley graph of $M$ with respect to $A$, in which vertices are elements from $M$ and, for every $m \in M$ and $a \in A$, we have an edge from $m$ to $m \cdot \varphi(a)$ labeled by the letter $a$. Thus, every $w_{n}$ labels a path from 1 to $\varphi\left(w_{n}\right)=\varphi(w)$ and one can extract a simple path from this path also starting in 1 and ending in $\varphi(w)$. If the sequence of labels of the edges in this simple path is $\left(a_{1}, \ldots, a_{k}\right)$, then the considered word $w_{n}$ can be written as $w_{n}=u_{0} a_{1} u_{1} \ldots a_{k} u_{k}$ for some words $u_{0}, \ldots, u_{k}$. The assumptions concerning the extracted simple path also implies that $k<|M|$ and $\varphi\left(a_{1} \ldots a_{k}\right)=\varphi(w)$. Since there are only finitely many simple paths in the Cayley graph of $M$, in infinitely many cases the extracted simple paths for words $w_{n}$ are the same. In this way we obtain a word $a_{1} \ldots a_{k}$, a label of a simple path from 1 to $\varphi(w)$, and a subsequence $\left(w_{n_{i}}\right)$ of the sequence $\left(w_{n}\right)$, such that $w_{n_{i}}=u_{i, 0} a_{1} u_{i, 1} \ldots a_{k} u_{i, k}$ for some appropriate words $u_{i, j}$. Now, by compactness, there is a strictly increasing sequence $\left(i_{\ell}\right)_{\ell}$ such that, for each $j=0, \ldots, k,\left(u_{i_{\ell}, j}\right)_{\ell}$ converges to some pseudoword $\bar{u}_{j}$. We thus obtain a final subsequence of $\left(w_{n}\right)_{n}$ converging to $w$ which shows that $w$ can be factorized as $w=\bar{u}_{0} a_{1} \bar{u}_{1} a_{2} \ldots a_{k} \bar{u}_{k}$. Hence, $v=a_{1} \ldots a_{k}$ is a finite subword of $w$ satisfying the required equality $\varphi(v)=\varphi(w)$.

Proposition 3. The pseudovariety $\mathrm{J}^{+}$is word-reducible (for $x \leq y$ ).

Proof. Let $u, v \in \bar{\Omega}_{A} \mathrm{M}$ be such that $\mathrm{J}^{+} \models u \leq v$, and let $\varphi: \bar{\Omega}_{A} \mathrm{M} \rightarrow M$ be a homomorphism to a finite monoid. By Lemma 2, there is a finite word $u^{\prime}=a_{1} a_{2} \ldots a_{n}$, with $a_{1}, a_{2}, \ldots, a_{n} \in A$, such that $u^{\prime}$ is a subword of $u$ satisfying $\varphi\left(u^{\prime}\right)=\varphi(u)$. Since $\mathrm{J}^{+} \models u^{\prime} \leq u$ and consequently $\mathrm{J}^{+} \models u^{\prime} \leq v$, there is a factorization $v=v_{0} a_{1} v_{1} a_{2} \ldots a_{n} v_{k}$ with $v_{0}, v_{1}, \ldots, v_{n} \in \bar{\Omega}_{A} \mathrm{M}$. Now, if we replace each $v_{i}$ by a finite word $v_{i}^{\prime}$ such that $\varphi\left(v_{i}^{\prime}\right)=\varphi\left(v_{i}\right)$, then we obtain the finite word $v^{\prime}=v_{0}^{\prime} a_{1} v_{1}^{\prime} a_{2} \ldots a_{n} v_{n}^{\prime}$. The constructed pair of words $u^{\prime}$ and $v^{\prime}$ have the following properties: $\varphi\left(u^{\prime}\right)=\varphi(u), \varphi\left(v^{\prime}\right)=\varphi(v)$, and $u^{\prime} \in \operatorname{Sub}\left(v^{\prime}\right)$, whence $\mathrm{J}^{+} \models u^{\prime} \leq v^{\prime}$.

The remainder of the paper presents several examples of pseudovarieties that are $\sigma$-equational but not $\sigma$-reducible, sometimes even not $\sigma^{\prime}$-reducible for a larger signature $\sigma^{\prime}$.

\section{Commutative Semigroups}

Our first negative example is that of the equational pseudovariety $\mathrm{Com}=$ $\llbracket x y=y x \rrbracket$ of all finite commutative semigroups. It is shown in [8] that Com is $(\omega-1)$-reducible, in fact for all finite systems of $(\omega-1)$-word equations. We show that it is not $\omega$-reducible, whence also not word reducible (for the equation $x=y$ ). Let Ab stand for the pseudovariety of all finite Abelian groups.

Theorem 4. No pseudovariety in the interval [Ab, Com] is $\omega$-reducible. 
Proof. Let $\mathrm{V}$ be a pseudovariety such that $\mathrm{Ab} \subseteq \mathrm{V} \subseteq$ Com. We exhibit a finite semigroup $S$, elements $s, t \in S$ and an onto continuous homomorphism $\varphi: \bar{\Omega}_{\{x, y\}} S \rightarrow S$ such that there is a Com-solution of the equation $x=y$ for the triple $(S, s, t)$ but no such solution exists in $\omega$-words. Let $X=\{x, y\}$ and $A=\{a, b\}$. We take $S$ to be the syntactic semigroup over the alphabet $A$ of the language $L=\left(\left(a^{2} b\right)^{2}\right)^{*} \cup\left(\left(a b^{2}\right)^{2}\right)^{*}$ and we denote by $[w]$ the syntactic class of a word $w \in\{a, b\}^{+}$. Let $s=\left[b a b^{2}\right]$, and $t=\left[a^{2} b a\right]$. A standard calculation shows that $s=b a b^{2}\left(\left(a b^{2}\right)^{2}\right)^{*}, t=\left(\left(a^{2} b\right)^{2}\right)^{*} a^{2} b a$, and

$$
\left[a b^{2}\right]^{\omega-1}=\left[a b^{2}\right], \quad\left[a^{2} b\right]^{\omega-1}=\left[a^{2} b\right] .
$$

The continuous homomorphism $\varphi$ is defined by letting $\varphi(x)=[a]$ and $\varphi(y)=$ $[b]$. Note that, for a word $w \in\{a, b\}^{+}$, the set $\varphi^{-1}([w])$ is the topological closure of $\eta^{-1}([w])$, where $\eta$ is the restriction of $\varphi$ to $X^{+}$, that is, essentially the syntactic homomorphism of the language $L$ up to the change of letters $x \leftrightarrow a, y \leftrightarrow b$.

We claim that there is no $\mathrm{V}$-solution of the equation $x=y$ for the triple $(S, s, t)$ in $\omega$-words. Let $u, v \in \bar{\Omega}_{X} S$ be pseudowords such that $\varphi(u)=s$ and $\varphi(v)=t$. The above description of the syntactic class $s$ shows that $u \in$ $\overline{y x y^{2}\left(\left(x y^{2}\right)^{2}\right)^{*}}=y x y^{2}\left\langle\left(x y^{2}\right)^{2}\right\rangle^{1}$, where $\langle w\rangle$ denotes the closed subsemigroup of $\bar{\Omega}_{X} \mathrm{~S}$ generated by $w$; similarly, we have $v \in\left\langle\left(x^{2} y\right)^{2}\right\rangle^{1} x^{2} y x$.

Let $\pi: \bar{\Omega}_{X} S \rightarrow \bar{\Omega}_{X}$ Ab be the natural continuous homomorphism, mapping each free generator to itself. It is well known that the profinite group $\bar{\Omega}_{X} \mathrm{Ab}$ is isomorphic with the product of two copies of the additive group $\hat{\mathbb{Z}}$ and we identify it with this product.

Note that, for $w \in \bar{\Omega}_{X} \mathrm{~S}$, since $\pi\left(w^{\omega}\right)$ is an idempotent, it is the identity element of the group $\hat{\mathbb{Z}} \times \hat{\mathbb{Z}}$. By induction on the construction of an $\omega$-word from the generators, it follows that $\pi\left(\Omega_{X}^{\omega} \mathrm{S}\right) \subseteq \mathbb{N} \times \mathbb{N}$, the reverse inclusion being obvious.

For each $w \in \bar{\Omega}_{X} \mathrm{~S}$, we let $\left(|w|_{x},|w|_{y}\right)=\pi(w)$. By the above discussion, there exist $\alpha, \beta \in \hat{\mathbb{Z}}$ such that

$$
|u|_{x}=2 \alpha+1,|u|_{y}=4 \alpha+3,|v|_{x}=4 \beta+3,|v|_{y}=2 \beta+1 .
$$

Assuming that $\alpha, \beta \in \mathbb{Z}$ and $\mathrm{Ab} \models u=v$, which entails $|u|_{x}=|v|_{x}$ and $|u|_{y}=|v|_{y}$, we obtain the system of equations $\beta=2 \alpha+1$ and $\alpha=2 \beta+1$, whose only integer solution is $\alpha=\beta=-1$. Hence, $u$ and $v$ cannot both be $\omega$-words, which establishes the claim. On the other hand, in view of the preceding calculations and (11), the pair $\left(y\left(x y^{2}\right)^{\omega-1},\left(x^{2} y\right)^{\omega-1} x\right)$ is a Vsolution of the equation $x=y$ for the triple $(S, s, t)$. Hence, $\mathrm{V}$ is not $\omega$ reducible.

\section{Groups}

We say that a pseudovariety $\mathrm{V}$ has infinite exponent if it satisfies no pseudoidentity of the form $x^{\omega+n}=x^{\omega}$, where $n$ is a positive integer. Given a set $\sigma$ of pseudowords, we let $\mathbf{H}_{\sigma}=\llbracket w=1: w \in \sigma \rrbracket$, which is a pseudovariety of groups.

Theorem 5. Let $\sigma$ be a set of binary implicit operations on the alphabet $X=\{x, y\}$ in which every element $w$ satisfies one of the following properties: 
(1) either $x^{3}$ or $y^{3}$ is a suffix of $w$;

(2) both $x y x$ and yxy are subwords of $w$.

Suppose further that the pseudovariety $\mathrm{H}_{\sigma}$ has infinite exponent. Then, no pseudovariety in the interval $\left[\mathrm{H}_{\sigma} \cap \mathrm{Ab}, \mathrm{H}_{\sigma}\right]$ is $\sigma \cup\{\omega\}$-reducible.

Proof. Let $\mathrm{H}$ be a pseudovariety in the interval $\left[\mathrm{H}_{\sigma} \cap \mathrm{Ab}, \mathrm{H}_{\sigma}\right]$. Note that $\mathrm{G} \models x^{\omega-1} y^{\omega} x^{2}=x$. We exhibit a semigroup $S$ and a pair of its elements $s, t$ such that $\left(x^{\omega-1} y^{\omega} x^{2}, x\right)$ is an $\mathrm{H}$-solution of the equation $x=y$ for the triple $(S, s, t)$, which has no $\mathrm{H}$-solution consisting of $\sigma \cup\{\omega\}$-words. We take $S$ to be the syntactic semigroup of the language $L=a^{2} a^{+} b^{+} a^{2}$ over the alphabet $A=\{a, b\}$. For each $w \in L$, a pair $(p, q) \in A^{*} \times A^{*}$ is a context of $w$, that is, $p w q \in L$, if and only if $p \in a^{*}$ and $q$ is the empty word. This means that all words from $L$ form one syntactic class $L=[w]$, for any $w \in L$, for instance for $w=a^{3} b a^{2}$. Since a pair $\left(a^{3} b a, 1\right)$ is a context of the word $a$ and it is not a context of any other word, we get $[a]=\{a\}$. Similarly, we can also see that $\left[a^{4}\right]=\left[a^{3}\right]$ and $\left[b^{2}\right]=[b]$.

Now, consider the onto continuous homomorphism $\hat{\varphi}: \bar{\Omega}_{X} S \rightarrow S$, which is the extension of the homomorphism $\varphi: X^{*} \rightarrow S$ uniquely given by $\varphi(x)=[a]$ and $\varphi(y)=[b]$. Further, we put $s=\hat{\varphi}\left(x^{\omega-1} y^{\omega} x^{2}\right)=[a]^{\omega-1}[b]^{\omega}[a]^{2}=\left[a^{3} b a^{2}\right]$ and $t=\hat{\varphi}(x)=[a]$, so that the pair $\left(x^{\omega-1} y^{\omega} x^{2}, x\right)$ is a $\mathrm{G}$-solution of the equation $x=y$ for the triple $(S, s, t)$, whence also an $\mathrm{H}$-solution. We show that there is no $\mathrm{H}$-solution of the equation $x=y$ for the triple $(S, s, t)$ in $\sigma \cup\{\omega\}$-words.

Suppose that $u, v \in \bar{\Omega}_{X} \mathrm{~S}$ are such that $\hat{\varphi}(u)=s, \hat{\varphi}(v)=t$ and $\mathbf{H} \models u=v$. Since $t=[a]=\{a\}$ implies $\varphi^{-1}(t)=\{x\}$, we have also $\hat{\varphi}^{-1}(t)=\{x\}$ and, consequently, $v=x$. Now, we see that $\varphi^{-1}(s)=\left\{x^{m} y^{n} x^{2} \mid m \geq 3, n \geq 1\right\}$, because $s=\left[a^{3} b a^{2}\right]=L$. The pseudoword $u$ must, therefore, be a limit of words from the set $\left\{x^{m} y^{n} x^{2} \mid m \geq 3, n \geq 1\right\}$; in particular, $u$ does not contain $y x y$ as a subword. Hence, $u$ is equal (as an element of $\bar{\Omega}_{X} \mathrm{~S}$ ) to a pseudoword of the form $x^{\alpha} y^{\beta} x^{2}$, where $\alpha, \beta \in \hat{\mathbb{N}} \backslash\{0\}$. Thus, $\mathrm{H}$ satisfies the pseudoidentity $x^{\alpha} y^{\beta} x^{2}=x$.

Now, suppose that $u$ is a $\sigma \cup\{\omega\}$-word. We claim that this assumption leads to a contradiction, namely that $\mathrm{H}_{\sigma}$ satisfies some identity of the form $x^{k+2}=x$, where $k \in \mathbb{N}$, which is contrary to the hypothesis that $\mathrm{H}_{\sigma}$ has infinite exponent and thereby concludes the proof. To prove the claim, consider an expression of $u$ as a $\sigma \cup\{\omega\}$-word. Since $u$ is not a word, such an expression must be of the form $u=u_{0} \psi(w) u_{1}$, where $u_{0}$ is another $\sigma \cup\{\omega\}$-word, $u_{1}$ is a word, $w \in \sigma \cup\left\{x^{\omega}\right\}$, and $\psi$ is a continuous endomorphism of $\bar{\Omega}_{X} \mathrm{~S}$. If $\left|u_{1}\right| \geq 2$, then $x^{2}$ is a suffix of $u_{1}$ and the claim holds since $\mathrm{H} \models u_{0} \psi(w)=u_{2}$ for some word $u_{2}$ which, upon identification of the variables $x$ and $y$, reduces the pseudoidentity $u=v$ to an identity of the form $x^{k+2}=x$. Hence, we may assume that $\left|u_{1}\right| \leq 1$, so that $\psi(w)$ must end with the letter $x$.

Let $z$ be the last letter of $w$, which entails that $x$ is the last letter of $\psi(z)$. Note that $w$ cannot be $x^{\omega}$ for, otherwise, $x^{3}$ would be a suffix of $u=x^{\alpha} y^{\beta} x^{2}$, which is clearly not the case. Suppose first that $w$ satisfies the condition (11) of the hypothesis, so that $z^{3}$ is a suffix of $w$. If both letters $x$ and $y$ appear in $\psi(z)$ then, since $\psi(z)$ ends with $x, y x$ is a subword of $\psi(z)$, so $y x y$ is a subword of $\psi\left(z^{2}\right)$, whence also of $\psi(w)$ and of $u$, which we know to be false. Hence, $\psi(z)$ must be of the form $x^{\gamma}$, with $\gamma \in \hat{\mathbb{N}} \backslash\{0\}$. Again, since $z^{3}$ is a 
suffix of $w$, it follows that $x^{3}$ is a suffix of $u$, which is not the case. It remains to consider the case where $w$ satisfies the condition (2) of the hypothesis. If $\psi(w)$ is a power of $x$, then again $x^{3}$ is a suffix $\psi(w)$, whence of $u$, which is false. Hence, both letters $x$ and $y$ intervene in $\psi(w)$. Since both $x y x$ and $y x y$ are subwords of $w$, it follows $y x y$ is a subword of $u$, which is false. This concludes the proof of the claim.

Of course, the dual of the theorem, where "suffix" is replaced by "prefix" in condition (1) is also valid. In case $\sigma$ is a singleton set, we may combine these two results to obtain a stronger result.

Corollary 6. Let $u \in \bar{\Omega}_{\{x, y\}} \mathrm{S}$ be a pseudoword such that the group pseudovariety $\mathrm{H}_{u}=\llbracket u=1 \rrbracket$ has infinite exponent. Then no pseudovariety in the interval $\left[\mathrm{H}_{u} \cap \mathrm{Ab}, \mathrm{H}_{u}\right]$ is $\{u, \omega\}$-reducible.

Proof. If any of the conditions (11), or its dual, or (2) of Theorem 5 is satisfied by $u$, then we may apply the theorem to obtain the desired non-reducibility property. Otherwise, up to exchanging variables, we may assume that $u$ is a pseudoword of the form $x^{m} y^{\alpha} x^{n}$, where $m, n \in\{1,2\}$. But then, substituting $y$ by $x^{\omega}$ in the pseudoidentity $u=1$, we see that $\mathrm{H}_{u} \models x^{m+n}=1$, which contradicts the assumption that $\mathrm{H}_{u}$ has infinite exponent.

In particular, the pseudovarieties $\mathrm{G}$ and $\mathrm{G}_{p}=\llbracket x^{p^{\omega}}=1 \rrbracket$, where $p$ is prime, are not $\omega$-reducible: take $\sigma=\left\{x^{\omega}\right\}$ for the first of these pseudovarieties and $\sigma=\left\{x^{p^{\omega}}\right\}$ for the latter, which is in fact not $\left\{\omega, p^{\omega}\right\}$-reducible. Many other examples can be considered. By [9, Theorem 3.2], every extensionclosed pseudovariety of groups is of the form $\mathrm{H}_{u}$ for some $u \in \bar{\Omega}_{\{x, y\}} \mathrm{S}$, and therefore Corollary 6 yields that, if nontrivial, then the pseudovariety $\mathrm{H}_{u}$ is not $\{u, \omega\}$-reducible. Note that, for the pseudovariety $\mathrm{G}_{\text {sol }}$ of all finite solvable groups, concrete pseudowords $u \in \bar{\Omega}_{\{x, y\}} \mathrm{S}$ such that $\mathrm{H}_{u}=\mathrm{G}_{\text {sol }}$ have been hard to construct, with arguments that depend on the classification of finite simple groups [15, 16]. Another important pseudovariety of the form $\mathrm{H}_{u}$ is $\mathrm{G}_{\text {nil }}$, of all finite nilpotent groups, where $u=\left[x,_{\omega} y\right]=\lim _{n \rightarrow \infty}\left[x,_{n} y\right]$, with the iterated commutator defined recursively by $[s, t]=s^{\omega-1} t^{\omega-1} s t$ and $[s, n+1 t]=[[s, n t], t]$. In this case, it is easy to see that one may apply Theorem 5 directly.

\section{Completely Regular semigroups}

The aim of this section is to prove that the pseudovariety $\mathrm{CR}=\llbracket x^{\omega+1}=$ $x \rrbracket$, consisting of all completely regular semigroups, is not $\omega$-reducible. Since our proof technique is similar to the case of groups, we consider the more general case of the pseudovarieties $\mathrm{CR}\left(\mathrm{H}_{\sigma}\right)=\mathrm{CR} \cap \overline{\mathrm{H}}_{\sigma}$ of all completely regular semigroups whose subgroups belong to $\mathrm{H}_{\sigma}$, where $\mathrm{H}_{\sigma}$ has infinite exponent and $\sigma$ satisfies some suitable combinatorial hypothesis to be specified below. Note that $\mathrm{CR}$ can be obtained as $\mathrm{CR}\left(\mathrm{H}_{\omega}\right)$, because $\mathrm{H}_{\omega}=\mathrm{G}$.

We say that a pseudoword $w$ has two disjoint occurrences of another pseudoword $u$ if there is a factorization of $w$ in which $u$ appears twice as a factor. A word $u$ is said to appear as a factor of $w$ within bounded distance from the end if some finite suffix of $w$ admits $u$ as a factor. 
Theorem 7. Let $\sigma$ be a set of binary implicit operations on the alphabet $X=\{x, y\}$ in which every element satisfies one of the following properties:

(1) either $x^{3}$ or $y^{3}$ is a suffix of $w$;

(2) each factor of length 4 of $w$ within bounded distance from the end has two disjoint occurrences in $w$.

Suppose further that the pseudovariety $\mathrm{H}_{\sigma}$ has infinite exponent. Then, no pseudovariety in the interval $\left[\mathrm{H}_{\sigma} \cap \mathrm{Ab}, \mathrm{CR}\left(\mathrm{H}_{\sigma}\right)\right]$ is $\sigma \cup\{\omega\}$-reducible.

Proof. Let $\mathrm{V}$ be an arbitrary pseudovariety from the interval $\left[\mathrm{H}_{\sigma} \cap \mathrm{Ab}, \mathrm{CR}\left(\mathrm{H}_{\sigma}\right)\right]$. We claim that the pseudoidentity $\left(x^{2} y\right)^{\omega-1}\left(x y^{2}\right)^{\omega}\left(x^{2} y\right)^{2}=x^{2} y$ is valid in CR. To prove the claim without invoking the general solution of the $(\omega-1)$-word problem for CR 20, 11, let $T$ be a finite completely regular semigroup and $\varphi: \bar{\Omega}_{X} \mathrm{~S} \rightarrow T$ be a continuous homomorphism. We let $p=\varphi(x)$ and $q=\varphi(y)$. Note that the elements $q p$ and $q^{2} p$ are $\mathcal{L}$-equivalent. Therefore, $\left(q^{2} p\right)^{\omega}$, an idempotent $\mathcal{L}$-equivalent to $q p$, is right neutral to $q p$. Thus we obtain $q p\left(q^{2} p\right)^{\omega}=q p$, where the left hand side can be written as $q\left(p q^{2}\right)^{\omega} p$. It follows that $\left(p^{2} q\right)^{\omega-1}\left(p q^{2}\right)^{\omega}\left(p^{2} q\right)^{2}=\left(p^{2} q\right)^{\omega-1}\left(p^{2} q\right)^{2}$, where the right hand side is equal to $p^{2} q$ in the completely regular semigroup $T$. Finally, since the pseudoidentity is valid in $\mathrm{CR}$, it is valid also in $\mathrm{V}$.

We proceed in a similar way as in the proof of Theorem 5. Let $S$ be the syntactic semigroup of the language $L=\left(a^{2} b\right)^{2}\left(a^{2} b\right)^{+}\left(a b^{2}\right)^{+}\left(a^{2} b\right)^{2}$ over the alphabet $A=\{a, b\}$. Note that each word $w$ from the language $L$ has a unique occurrence of the factor $b^{2} a^{2}$. Hence, if two words from $L$ overlap, then one is a suffix of the other. Therefore, for each $w \in L$, a pair $(p, q) \in A^{*} \times A^{*}$ is a context of $w$, if and only if $p \in\left(a^{2} b\right)^{*}$ and $q$ is the empty word. This means that $L$ forms one syntactic class. Since a pair $\left(\left(a^{2} b\right)^{3}\left(a b^{2}\right)\left(a^{2} b\right), 1\right)$ is a context of the word $a^{2} b$ and it is not a context of any other word, we get $\left[a^{2} b\right]=\left\{a^{2} b\right\}$. One can also check that $\left[a^{2} b\right]^{4}=\left[a^{2} b\right]^{3}$ and $\left[a b^{2}\right]^{2}=\left[a b^{2}\right]$.

For $X=\{x, y\}$, we consider an onto continuous homomorphism $\hat{\varphi}$ : $\bar{\Omega}_{X} S \rightarrow S$, which is the extension of $\varphi: X^{*} \rightarrow S$ uniquely given by $\varphi(x)=[a]$ and $\varphi(y)=[b]$. We put

$$
s=\hat{\varphi}\left(\left(x^{2} y\right)^{\omega-1}\left(x y^{2}\right)^{\omega}\left(x^{2} y\right)^{2}\right)=\left[a^{2} b\right]^{\omega-1}\left[a b^{2}\right]^{\omega}\left[a^{2} b\right]^{2}=\left[\left(a^{2} b\right)^{3}\left(a b^{2}\right)\left(a^{2} b\right)^{2}\right]
$$

and $t=\hat{\varphi}\left(x^{2} y\right)=\left[a^{2} b\right]$. Assume that there are $\sigma \cup\{\omega\}$-words $u, v \in \Omega_{X}^{\sigma \cup\{\omega\}} \mathrm{S}$ such that $\hat{\varphi}(u)=s, \hat{\varphi}(v)=t$ and $\mathrm{V} \models u=v$.

We have $t=\left[a^{2} b\right]=\left\{a^{2} b\right\}$, which implies $(\hat{\varphi})^{-1}(t)=\left\{x^{2} y\right\}$. So, we have $v=x^{2} y$. Furthermore, we see that

$$
\varphi^{-1}(s)=\left\{\left(x^{2} y\right)^{m}\left(x y^{2}\right)^{n}\left(x^{2} y\right)^{2} \mid m \geq 3, n \geq 1\right\},
$$

because $s=\left[\left(a^{2} b\right)^{3}\left(a b^{2}\right)\left(a^{2} b\right)^{2}\right]=L$. The pseudoword $u$ must be a limit of words from the set $\varphi^{-1}(s)$. Therefore, $u$ is of the form $\left(x^{2} y\right)^{\alpha}\left(x y^{2}\right)^{\beta}\left(x^{2} y\right)^{2}$, where $\alpha, \beta \in \hat{\mathbb{N}}$.

Consider an expression of $u$ as a $\sigma \cup\{\omega\}$-word. Since $u$ is not a word, such an expression must be of the form $u=u_{0} \psi(w) u_{1}$, where $u_{0}$ is another $\sigma \cup\{\omega\}$-word, $u_{1} \in X^{*}$ is a word, $w \in \sigma \cup\left\{x^{\omega}\right\}$, and $\psi$ is a continuous endomorphism of $\bar{\Omega}_{X} \mathrm{~S}$. We claim that $\left|u_{1}\right| \geq 4$, in which case we are able to proceed as in the proof of Theorem 5 . 
Assume for a moment, that $\left|u_{1}\right|<4$. In other words, we have $u_{1} \in$ $\left\{1, y, x y, x^{2} y\right\}$. First, we discuss the case when $w$ satisfies condition (1). Let $z \in X$ be the last letter of $w$, so that $z^{3}$ is a suffix of $w$. We can see that $|\psi(z)| \geq 2$, because $u$ does not contain a cube of a letter as a factor. In case $|\psi(z)|=2$, one can easily check that none of the possible alternatives $\psi(z)=x^{2}, \psi(z)=x y, \psi(z)=y x$ or $\psi(z)=y^{2}$ can hold as $\psi(z) \psi(z) u_{1}$ is a suffix of $y x^{2} y x^{2} y$. Thus, we have $|\psi(z)| \geq 3$ and, therefore, $\left|\psi\left(z^{3}\right) u_{1}\right| \geq 9$. This means that $(\psi(z))^{3}$ contains as a factor the word $y^{2} x^{2}$. Since this factor has length 4 and the length of $\psi(z)$ is at least 3 , we deduce that $y^{2} x^{2}$ is even a factor of $\psi(z)^{2}$. However, in such a case the factor $y^{2} x^{2}$ has at least two disjoint occurrences in $\psi(z)^{3}$, which is not possible, as $u$ contains just one occurrence of the factor $y^{2} x^{2}$.

Now, assume that $w$ satisfies condition (21) and recall that $w$ is not a word. As in the first case, under the assumption that $\left|u_{1}\right|<4$, one can show that the word $y^{2} x^{2}$ is a factor of $\psi(w)$. This means that it is a factor of some $\psi\left(w^{\prime}\right)$ where $w^{\prime}$ is a factor of $w$ of length 4 within bounded distance from the end. Since $w$ satisfies condition (2), we deduce that $\psi(w)$ contains another disjoint occurrence of $y^{2} x^{2}$, which is a contradiction. This completes the proof of the claim that $\left|u_{1}\right| \geq 4$.

We can reformulate the previous claim as follows. The pseudoword $u$ is a product of a certain $\sigma \cup\{\omega\}$-word $u^{\prime}$ of the form $\left(x^{2} y\right)^{\alpha}\left(x y^{2}\right)^{\beta} x^{2}$ and the finite word $y x^{2} y$. Next, we consider the continuous homomorphism $\varphi$ : $\bar{\Omega}_{X} \mathrm{~S} \rightarrow \bar{\Omega}_{X} \mathrm{~S}$ given by $\varphi(x)=\varphi(y)=x$. Since $\mathrm{V} \models u=v$, we obtain $\mathrm{V} \models \varphi(u)=\varphi(v)$. Since $\mathrm{H}_{\sigma} \cap \mathrm{Ab} \subseteq \mathrm{V} \subseteq \mathrm{CR}\left(\mathrm{H}_{\sigma}\right)$, the pseudoidentity $\varphi(u)=\varphi(v)$ must be valid in $\mathrm{H}_{\sigma} \cap \mathrm{Ab}$. The prefix of the left hand side, corresponding to the prefix $\varphi\left(u^{\prime}\right)$ of $\varphi(u)$, is equivalent over $\mathrm{H}_{\sigma} \cap \mathrm{Ab}$ to $x^{k}$ for some non-negative integer $k$. This means that, over $\mathrm{H}_{\sigma} \cap \mathrm{Ab}$, the pseudoidentity $\varphi(u)=\varphi(v)$ is equivalent to a certain identity $x^{k} x^{4}=x^{3}$ for some non-negative integer $k$. However such pseudoidentity is not valid in $\mathrm{H}_{\sigma} \cap \mathrm{Ab}$, because $\mathrm{H}_{\sigma} \cap \mathrm{Ab}$ and $\mathrm{H}_{\sigma}$ satisfy the same unary pseudoidentities and the pseudovariety $\mathrm{H}_{\sigma}$ has infinite exponent, a contradiction.

This means that the equation $x=y$ does not have a $\mathrm{V}$-solution for the triple $(S, s, t)$ consisting of $\sigma \cup\{\omega\}$-words. On the other hand, we saw that the equation $x=y$ has a $\mathrm{V}$-solution consisting of $(\omega-1)$-words for the same triple $(S, s, t)$. We conclude that $\mathrm{V}$ is not $\sigma \cup\{\omega\}$-reducible.

Note that Theorem 7 has some overlap with Theorem 5 but does not quite supersede it. We have not succeeded in finding an analog of Corollary 6 for Theorem 7 .

Examples of application of Theorem 7 include $\mathrm{CR}, \mathrm{CR}\left(\mathrm{G}_{p}\right)$, and $\mathrm{CR}\left(\mathrm{G}_{\mathrm{nil}}\right)$, where $\sigma=\{u\}$, respectively with $u=x^{\omega}, u=x^{p^{\omega}}$, and $u=\left[x,_{\omega} y\right]$.

Another example is obtained by taking $u=\mu^{\omega}(x)=\lim _{n \rightarrow \infty} \mu^{n !}(x)$, where $\mu$ is the Prouhet-Thue-Morse substitution, defined as the endomorphism of $\{x, y\}^{+}$such that $\mu(x)=x y$ and $\mu(y)=y x$. The length of the word $\mu^{n}(x)$ is $2^{n}$. Hence, by identification of the variables $x$ and $y$, we conclude that $\mathrm{H}_{u} \subseteq \mathrm{G}_{2}$. The reverse inclusion is a particular case of a general result, namely [4, Proposition 5.6]. It is well know that each $\mu^{n}(x)$ is a cube-free word, in the sense that no nonempty factor is a cube 21. Hence, the same is true of the pseudoword $u$. On the other hand, $u$ is a regular element of the 
semigroup $\bar{\Omega}_{A} \mathrm{~S}$, which entails that it satisfies condition (2) of Theorem 7 . Thus, $\mathrm{CR}\left(\mathrm{G}_{2}\right)$ is neither $\{u, \omega\}$ nor $\left\{2^{\omega}, \omega\right\}$-reducible.

\section{ACKNOWLEDGMENTS}

The first author acknowledges partial funding by CMUP (UID/MAT/ 00144/2013) which is funded by FCT (Portugal) with national (MCTES) and European structural funds (FEDER) under the partnership agreement PT2020. The second author was supported by Grant 15-02862S of the Grant Agency of the Czech Republic.

\section{REFERENCES}

1. D. Albert, R. Baldinger, and J. Rhodes, The identity problem for finite semigroups (the undecidability of), J. Symbolic Logic 57 (1992), 179-192.

2. J. Almeida, Finite semigroups and universal algebra, World Scientific, Singapore, 1995, English translation.

3. , Hyperdecidable pseudovarieties and the calculation of semidirect products, Int. J. Algebra Comput. 9 (1999), 241-261.

4. _ Dynamics of finite semigroups, Semigroups, Algorithms, Automata and Languages (Singapore) (G. M. S. Gomes, J.-E. Pin, and P. V. Silva, eds.), World Scientific, 2002, pp. 269-292.

5. _ Profinite semigroups and applications, Structural theory of automata, semigroups and universal algebra (New York) (V. B. Kudryavtsev and I. G. Rosenberg, eds.), Springer, 2005, pp. 1-45.

6. J. Almeida, J. C. Costa, and M. Zeitoun, Complete reducibility of systems of equations with respect to $R$, Portugal. Math. 64 (2007), 445-508.

7. L Reducibility of pointlike problems, Semigroup Forum (2015), DOI: 10.1007/s00233-015-9769-2.

8. J. Almeida and M. Delgado, Tameness of the pseudovariety of abelian groups, Int. J. Algebra Comput. 15 (2005), 327-338.

9. J. Almeida, S. Margolis, B. Steinberg, and M. Volkov, Characterization of group radicals with an application to mal'cev products, Illinois J. Math. 54 (2010), 199-221.

10. J. Almeida and B. Steinberg, On the decidability of iterated semidirect products and applications to complexity, Proc. London Math. Soc. 80 (2000), 50-74.

11. J. Almeida and P. G. Trotter, The pseudoidentity problem and reducibility for completely regular semigroups, Bull. Austral. Math. Soc. 63 (2001), 407-433.

12. J. Almeida and P. Weil, Profinite categories and semidirect products, J. Pure Appl. Algebra 123 (1998), 1-50.

13. C. J. Ash, Inevitable graphs: a proof of the type II conjecture and some related decision procedures, Int. J. Algebra Comput. 1 (1991), 127-146.

14. K. Auinger and B. Steinberg, On the extension problem for partial permutations, Proc. Amer. Math. Soc. 131 (2003), 2693-2703.

15. T. Bandman, G.-M. Greuel, F. Grunewald, B. Kunyavskiı̌, G. Pfister, and E. Plotkin, Two-variable identities for finite solvable groups, C. R. Acad. Sci. Paris Sér. I Math. 337 (2003), 581-586.

16. J. N. Bray, J. S. Wilson, and R. A. Wilson, A characterization of finite soluble groups by laws in two variables, Bull. London Math. Soc. 37 (2005), 179-186.

17. J. C. Costa and C. Nogueira, Complete reducibility of the pseudovariety LSl., Int. J. Algebra Comput. 19 (2009), no. 2, 247-282.

18. S. Eilenberg, Automata, languages and machines, vol. A, Academic Press, New York, 1974.

19. Automata, languages and machines, vol. B, Academic Press, New York, 1976.

20. J. Kad'ourek and L. Polák, On the word problem for free completely regular semigroups, Semigroup Forum 34 (1986), 127-138.

21. M. Lothaire, Combinatorics on words, Addison-Wesley, Reading, Mass., 1983. 
22. V. A. Molchanov, Nonstandard characterization of pseudovarieties, Algebra Universalis 33 (1995), 533-547.

23. J.-E. Pin, Varieties of formal languages, Plenum, London, 1986, English translation.

24. _ Syntactic semigroups, Handbook of Formal Languages (G. Rozenberg and A. Salomaa, eds.), Springer, 1997.

25. J.-E. Pin and P. Weil, A Reiterman theorem for pseudovarieties of finite first-order structures, Algebra Universalis 35 (1996), 577-595.

26. _ Polynomial closure and unambiguous product, Theory Comput. Syst. 30 (1997), 383-422.

27. J. Reiterman, The Birkhoff theorem for finite algebras, Algebra Universalis 14 (1982), $1-10$.

28. J. Rhodes and B. Steinberg, The q-theory of finite semigroups, Springer Monographs in Mathematics, Springer, 2009.

CMup, Dep. Matemática, Faculdade de Ciências, Universidade do Porto, Rua do Campo Alegre 687, 4169-007 Porto, Portugal

E-mail address: jalmeida@fc.up.pt

Dept. Of Mathematics and Statistics, Masaryk University, KotláR̆ská 2, 61137 Brno, Czech Republic

E-mail address: klima@math.muni.cz 